\title{
Gutachterinnen und Gutachter der KZfSS 2003/2004
}

An der Begutachtung von Manuskripten, die der Zeitschrift im Zeitraum zwischen dem 1. Juli 2003 und dem 30. Juni 2004 eingereicht wurden, haben die folgenden Kolleginnen und Kollegen mitgewirkt. Wir danken ihnen sehr herzlich für diese aktive Beteiligung an der Entstehung der Zeitschrift.

Abraham, Martin (Leipzig) Albert, Hans (Heidelberg) Albrecht, Clemens (Koblenz) Andre $\beta$, Hans-Jürgen (Köln) Barlösius, Eva (Berlin) Baurmann, Michael (Düsseldorf) Bette, Karl-Heinrich (Darmstadt) Blasius, Jörg (Bonn) Blossfeld, Hans-Peter (Bamberg) Bös, Mathias (Marburg) Büchel, Felix (Berlin) Buchmann, Marlis (Zürich) Dollase, Rainer (Bielefeld) Ebbinghaus, Bernhard (Mannheim) Eifler, Stefanie (Bielefeld) Emrich, Eike (Frankfurt a.M.) Fuchs, Marek (Kassel) Geißler, Rainer (Siegen) Gergs, Hans-Joachim (Nürnberg) Glatzer, Wolfgang (Frankfurt a.M.) Haipeter, Thomas (Gelsenkirchen) Halfmann, Jost (Dresden) Hassel, Anke (Köln) Heinze, Rolf G. (Bochum) Hermann, Dieter (Heidelberg) Hill, Paul Bernhard (Aachen) Hillmert, Steffen (Berlin) Holtappels, Günter (Dortmund) Hornbostel, Stefan (Jena) Kappelhoff, Peter (Wuppertal) Klages, Helmut (Speyer) Klein, Thomas (Heidelberg) Kneer, Georg (Schwäbisch Gmünd) Knoblauch, Hubert (Berlin) Konietzka, Dirk (Rostock) König, Thomas (Konstanz) Kühnel, Steffen-M. (Göttingen) Kunczik, Michael (Mainz) Lamnek, Siegfried (Eichstätt)

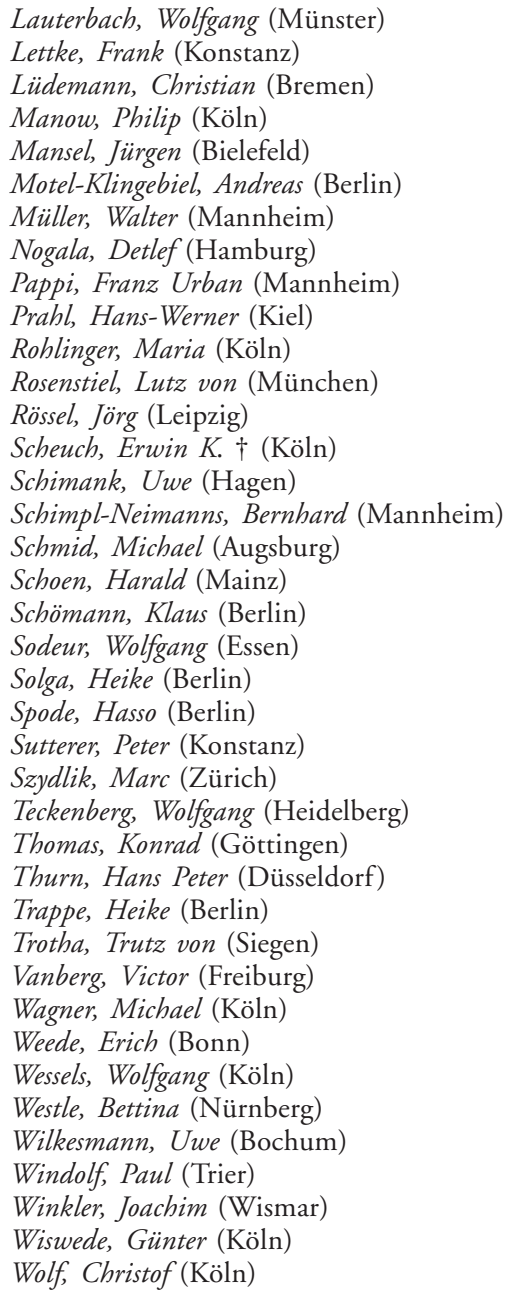

